\title{
A multi-functional design approach and proposed figure of merits for solid state lighting systems
}

\author{
Muhammed Nasir Inan ${ }^{*}$ and Mehmet Arik
}

\author{
* Correspondence: \\ nasir.inan@ozu.edu.tr \\ Department of Mechanical \\ Engineering, Ozyegin University, \\ Nisantepe District, Orman Street, \\ 34794 Istanbul, Turkey
}

\begin{abstract}
Energy has been identified as one of the most important problems during the last few years. One of energy efficient technologies may reduce consumed energy in buildings from 20 percent to less than 5 percent. A novel lighting technology has recently been evolved as light emitting diodes (LEDs) that can be over 7-10 times more efficient than conventional-old incandescent lamps. Therefore, LED lighting systems have been rapidly replacing conventional energy-hungry lighting products like incandescent lamps and more recently environmentally hazardous fluorescent lamps. While LEDs may produce large amounts of lumens, they are solid state based technologies similar to computer chips so they have to be kept cool at certain chip junction temperature. The demand for high light output LED systems lead to significant heat generation rates, so that higher heat fluxes result in elevated junction temperatures on LED chips in SSL lighting systems. Moreover, the changes on the junction temperature strongly impact the reliability, lifetime, light output and quality of the light. Because of their simplicity, reliability, low cost and silent operation, passive air-cooling systems are preferred in LED lamps. Passive metal based heat sinks are the main cooling components of typical LED lamps serves for both LEDs and driver electronics. Heat is dissipated generally from finned surfaces to ambient air with primarily convection mechanism and partially radiation. But it requires a large surface area and weight under the limitations of the standardized lamps. Thus, the optimization of the heat sink in an LED system is crucial. Developing figure of merits (FOM) is very important for designers and researchers to find the most optimal solution accounting for critical metrics such as size, weight, cost and performance. In the present study, thermal, electrical and optical experimental results of various commercial A-line LED lamps are investigated and a number of FOMs are proposed based on the performance, size and weight. Proposed FOMs aim to evaluate different aspects by combining a number of performance metrics. Results show that one can combine and analyze multi-purpose design parameters for thermal, electrical and optical performances and manufacturing for engineers and consumers.
\end{abstract}

Keywords: LED; Thermal performance; Optical performance; Passive cooling; Heat sink; Figure of merit

\section{Background}

Energy is one of the top 10 challenges for human being as cited by Nobel prize laureate Richard Errett Smalley in 2001 [1]. Therefore during the last decade, there has been significant amount of effort devoted for a wide range of energy research including new resources, new methods of generation and energy efficiency. Lighting represents

\section{Springer}

(c) 2014 Inan and Arik; licensee Springer. This is an Open Access article distributed under the terms of the Creative Commons Attribution License (http://creativecommons.org/licenses/by/4.0), which permits unrestricted use, distribution, and reproduction in any medium, provided the original work is properly credited. 
approximately $16 \%$ of residential and $20 \%$ of commercial building electricity consumption [2]. Researchers developed technologies such as fluorescent lamps that has over four times of comparable incandescent lamps, and they have been widely used during the last four decades. LEDs are rather recent and the most efficient lighting technologies started taking its place in general lighting applications during the last decade and it promises even more energy savings than fluorescent lamps.

While LEDs can provide superior optical performances at low energy consumptions, they pose significant thermal challenges due to inherit solid-state device technology based on light-generating active layers. Interest in passive cooling of high power LED lamps is increasing with the high luminosity demand though their bulky size and weight. Metal based heat sinks are widely used passive cooling components for the last few decades since the cooling of electronics started to become a major challenge for engineers. Besides the simplicity of fabrication without the requirement of a high-tech manufacturing facility, mature industrial utilities keep heat sink production low priced on this long-experienced field. This was also driven by the electronics industry while the performance has been increasing, size and cost has been shrinking. Heat sink requirements are changing by the cooling needs of different electronics with their various size, weight and operational standards. Heat sinks used in LED lamps are not only cooling the system but also help to assemble electronics and optical components. Complex task of a heat sink design includes also the aesthetic side like thermal, optical and mechanical aspects to address the user's expectations to replace conventional lighting technologies.

Investigations on heat sink geometry with an optimization goal tend on the parameters like fin spacing, height, thickness, and material are seen in a number of studies [3-5]. The study [6] with the figure of merit of thermal performance related with the weight of heat sink gives an idea about the direction of the future research interests as:

$$
\mathrm{FOM}=\frac{1}{\mathrm{Rxm}}
$$

However, this expression accounts for thermal resistance and mass, while lighting products demand more than only two critical design and performance metrics. Complexity of combining various design perspectives with the development of new approaches for LED lamps is also related to the advancement in production technology development. The pursuit of extending heat sink surface area of standardized high power LED lamps may be considered well, since it means higher effort and price. Global lighting standards driven footprint area and volumetric dimensions should always be considered as a major design rule. Heat sinks should be designed cleverly for an effective utilization of both convective and radiative heat removal for a constrained weight and size. The necessity of a very low conductive thermal resistance is correlated with the materials besides the design, weight and price. Since multi-task design of various engineering fields is rather complicated, it is not sufficient to evaluate the LED lamps with the limited parameters such as standalone thermal, optical or electrical. In order to prevent the confusion, new FOMs are required to be developed for the multi-aspect performance of LED lamps. It is also very important to bring thermal, electrical and optical engineers together and to improve their design by understanding how it affects other components, when designing a complex system. This study offers several FOMs to enable the interpretation for the desired issues. Thermal and 
optical experimental test results of commercial A-line LED bulbs are presented based on the FOMs with a composition of different design approaches. Those commercial lamps are selected from off-the-shelf systems and there is no specific reason to choose for a particular brand. To avoid advertisement, no brand name is mentioned in this study. Beyond the designers and engineers, proposed FOMs also attempt to clarify consumer's confusion for the thermal side directly related to reliability and life time by combining with the optical, electrical, geometric and weight parameters.

\section{Methods}

Several commercial A-line LED bulbs from world's leading lighting vendors are investigated. Tested bulbs with various passive thermal solutions are entitled with the names starting from bulb-A to bulb-G. Chosen bulbs have different input powers, luminous fluxes, weights and sizes due to their designs. They are all omni-directional bulbs and have similar angular light intensity distributions. Only exception is bulb-G with a higher forward intensity which can be understood from its diffuser shape. They are carefully chosen to compare the opto-thermal performances of different cooling approaches from simple heat sink to external finned complex heat sink and liquid cooling approaches. The results display the total system performance of the tested bulbs, but it still enables the interpretation of cooling components. Some of the lamps have different electrical power inputs and light outputs with the same cooling component (Bulb E and Bulb F) to indicate their performances under different operating conditions. Information about the cooling approaches and heat sink designs of the bulbs and input powers given by manufacturers are shown in Table 1 .

Bulbs are tested in the air-tight setup created for the experimental study presented in Figure 1. The enclosure is designed with a plexiglas material, and it had a size of $120 \times 80 \times 90(\mathrm{~cm})$ to avoid any external air flow. A standard bulb base is used to screw Edison base lamps. No insulated ceiling structure is used here to simplify the testing procedure but still provides adequately accurate results.

Test set-up is prepared that A-line bulbs are placed to mimic a real life application. Temperature in the enclosure is constantly monitored and did not change during the experiments. A typical thermal experiment took place over 2 hours and steady state temperature measurements have been performed. Optical experiments have been performed at Ozyegin University EVATEG optical laboratory in an integrated sphere (Sphere Optics). Each experiment has been performed at least two times for repeatability and reproducibility, as well as reducing the uncertainty in the readings.

Table 1 Input powers and cooling approaches of test vehicles

\begin{tabular}{lll}
\hline & Input power $(\mathbf{W})$ & Cooling approach $\boldsymbol{\&}$ heat sink design \\
\hline Bulb A & 17 & Passive cooling with external fins \\
Bulb B & 9.5 & Passive cooling with small heat sink \\
Bulb C & 13.5 & Chimney effect with internal and external fins \\
Bulb D & 9 & Passive cooling with large external fins \\
Bulb E & 8 & Passive liquid cooling with external fins \\
Bulb F & 12 & Passive liquid cooling with external fins \\
Bulb G & 10 & Passive cooling with external fins \\
\hline
\end{tabular}




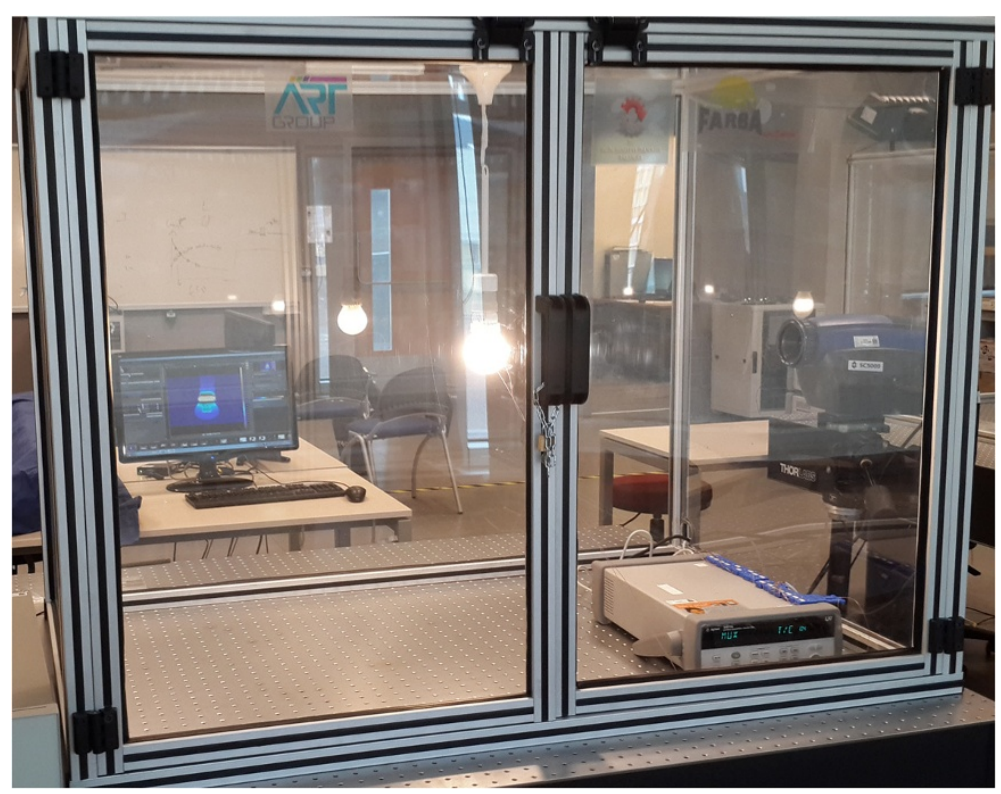

Figure 1 Experimental setup. Thermocouples are used to measure local temperatures and correlate emissivity

T-type thermocouples with a measurement error of $\pm 1^{\circ} \mathrm{C}$ are used in the experiments for temperature measurements. For the infrared camera (IR) thermal measurements, surface emissivities are calibrated with three distinctly located thermocouple readings. An Agilent data acquisition system is used in experiment with an accuracy of $0.1^{\circ} \mathrm{C}$ for temperature readings and 1\% for voltage readings. Two thermocouples were placed on lamp glass dome, heat sink base and heat sink area near to the Edison base to verify the temperature measurements by comparing with each other to increase the confidence in the emissivity correlation. They are attached to the surfaces with an RTV silicone to obtain a reliable contact. Intimate contact of thermocouples to the surfaces is assured by applying controlled pressure. Total system accuracy is adequate enough that no significant deviation for emissivity calibration can occur for the thermal imaging. The error of IR Camera (FLIR SC 5000) is $\pm 1^{\circ} \mathrm{C}$ [7]. IR images of the tested lamps are shown in Figure 2.

Optical measurements were done in a scientific integrating sphere with a diameter of $2 \mathrm{~m}$. Figure 3 presents the preparation stage of the optical measurements in the sphere for luminous flux, color rendering index (CRI), correlated color temperature (CCT) and other optical values. Optical results are obtained, when the heat sinks reached to the steady state temperatures as measured earlier with IR camera and data acquisition system. As expected both temperature and lumen output has reached at the steady state value. Steady state decision for temperature was less than $\pm 0.05 \mathrm{~K}$ variation in 5 minutes of data collection. Integrated sphere is calibrated to the reference bulb before each measurement. Optical results of test samples are shown in Table 2.

Surface area calculations are taken from CAD models for each lamp. Weight is also experimentally measured for combined performance analysis based on the new FOMs. Uncertainty in the weight measurements is less than $1 \%$. Real powers of lamps are measured with an Agilent digital storage oscilloscope. Results for the surface area, weight and input power are shown in Table 3. 


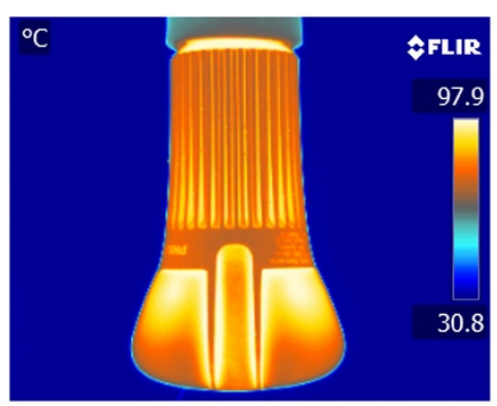

Bulb-A

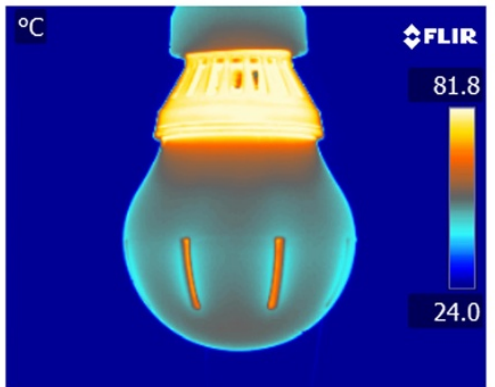

Bulb-C

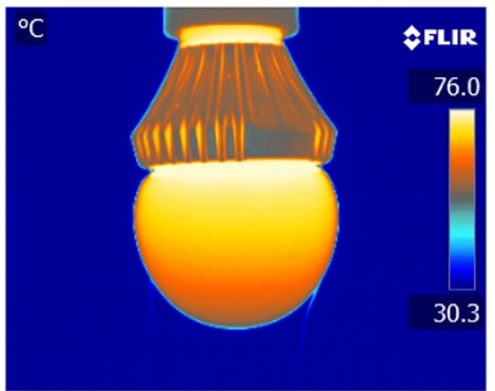

Bulb-E

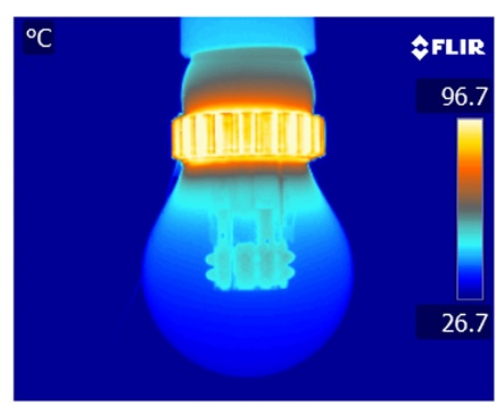

Bulb-B

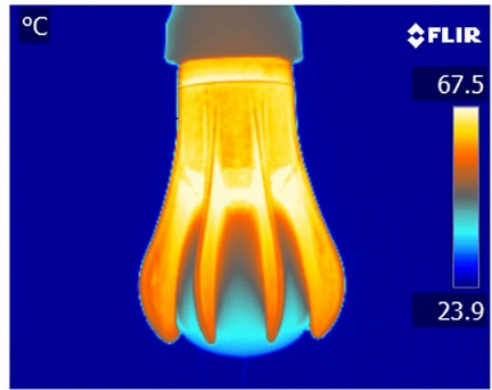

Bulb-D

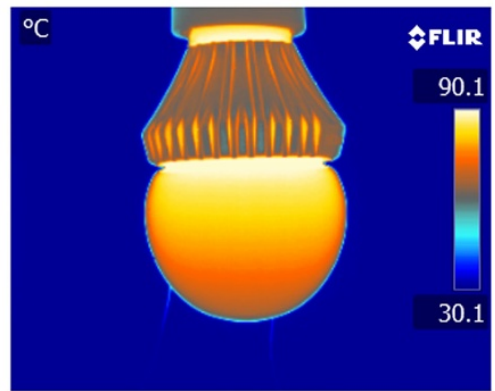

Bulb-F

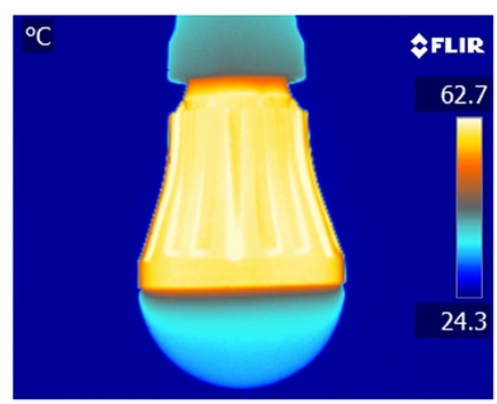

Bulb-G

Figure 2 IR images of tested bulbs.

\section{Results and discussions}

FOMs are produced from the analysis of the experimental results based on thermal resistance, luminous efficacy, opto-thermal and multi-aspect performances. These FOMs are presented in Table 4 to provide a common perspective for researchers and consumers.

Thermal resistance is one of the most critical parameters to evaluate the thermal performance of a cooling system. It is important to know the amount of heat that heat sink 


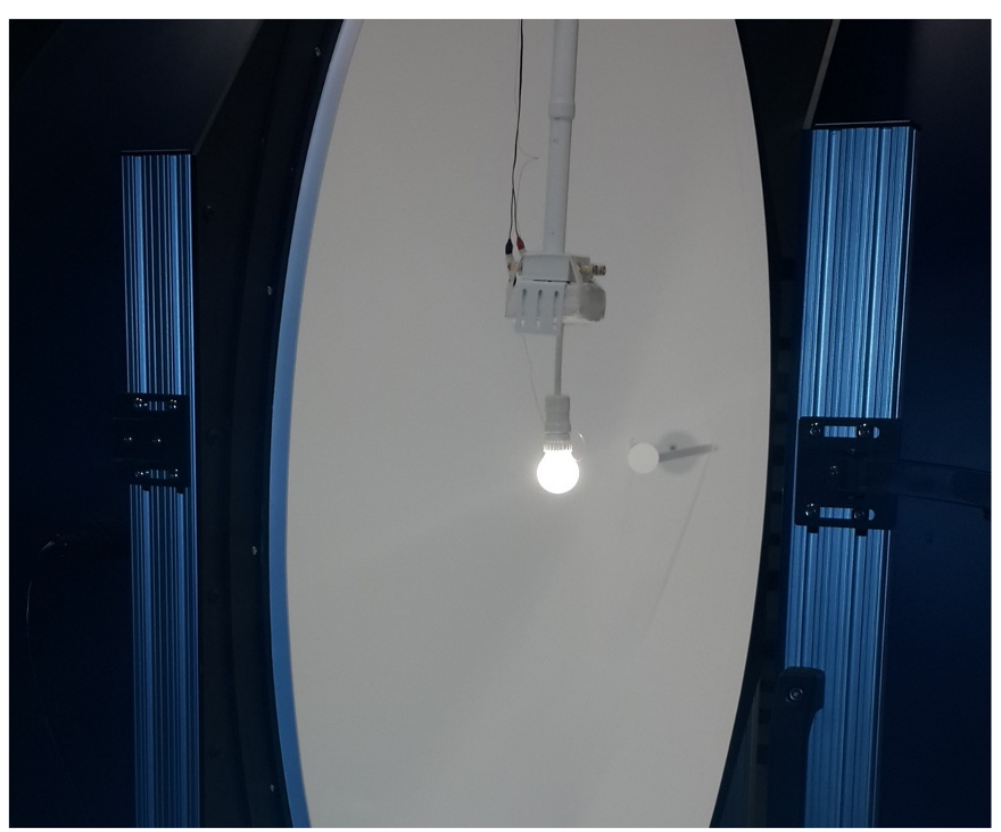

Figure 3 Integrated sphere. Calibrated before all measurements.

is capable of removing per surface area. It is usually assumed that a heat sink with a very intricate surface and low thermal resistance needs a long process to be manufactured. Low thermal resistance alone may not be enough to select for a heat sink because of the unacceptable time and energy consumption required during manufacturing or the manufacturing capability requirements. Target is the maximum heat removal with the simplest heat sink and the minimal use of material. From consumer's point of view, low weight is primarily preferred, since they are used to have low or no-weight products of traditional lighting technologies. Risk of injury by falling down of a ceiling lamp is another fact that needs to be considered in lighting systems. Derivations of this study are produced based on the mass of LED bulbs and surface area of heat sinks. $\mathrm{FOM}_{\mathrm{R}, \mathrm{m}}$ represents the product of the thermal resistance and the mass of bulb. $\mathrm{FOM}_{R, \mathrm{~A}}$ represents the product of the thermal resistance and the surface area of heat sink, that indicates how efficiently a heat sink removes heat to ambient air based on surface area. While most of the heat is through heat sink removed, it is a common practice to analyze the heat dissipation based on surface area of heat sink. $\mathrm{FOM}_{\mathrm{R}, \mathrm{m}, \mathrm{A}}$ is the final product of the thermal resistance, mass and surface area. Basic thermal resistance calculation used for the performance analysis of the tested bulbs is;

$$
\mathrm{R}=\frac{\mathrm{T}_{\max }-\mathrm{T}_{\mathrm{amb}}}{\mathrm{q}}
$$

where $T_{\max }$ is the maximum heat sink surface temperature measured with IR camera, $\mathrm{T}_{\mathrm{amb}}$ is the ambient temperature measured with thermocouples and $\mathrm{q}$ is the heat flow rate calculated as

$$
\mathrm{q}=\mathrm{P}-\Phi_{e}
$$


Table 2 Optical results of tested samples

\begin{tabular}{llll}
\hline & Luminous flux $(\mathbf{I m})$ & CCT (K) & CRI \\
\hline Bulb-A & 1244,0 & 2633 & 80,9 \\
Bulb-B & 858,6 & 2723 & 80,8 \\
Bulb-C & 913,3 & 2986 & 81,0 \\
Bulb-D & 551,5 & 6535 & 71,9 \\
Bulb-E & 545,0 & 2642 & 85,9 \\
Bulb-F & 855,0 & 2772 & 79,9 \\
Bulb-G & 793,0 & 6483 & 85,0 \\
\hline
\end{tabular}

where $\mathrm{P}$ is input power measured with oscilloscope and $\Phi_{\mathrm{e}}$ is radiant flux measured with integrated sphere. Comparison of tested bulbs based on the derivations of thermal resistance can be seen in Figures 4 and 5.

Bulb-B has the highest thermal resistance as seen in Figure 4 due to its small heat sink. Bulb-F indicates a decrease in thermal resistance with the increasing input power compared to bulb-E. Not only different LEDs and electronics components enhance the thermal performance, but also the increasing surface temperature of a heat sink simply increases the heat transfer coefficients and enhances the thermal performance. Bulb-A with the very high heat sink temperature and effective cooling design has the lowest thermal resistance. Bulb-G follows bulb-A based on thermal resistance. Bulb-G and bulb-D have the best results due to the thermal resistance based on mass. This shows the effective heat removal of simple heat sink with large surface area and light-weight external finned heat sink. Bulb-E and bulb-F with liquid cooling have the worst results on this perspective because of the large weight caused by liquid inside. Despite the fact that bulb-E and bulb-F have both high thermal resistances and mass based thermal resistances, liquid inside the dome prevents the hot spots on LEDs which is important for reliability. Heat transfer in dome direction is another benefit of liquid cooling inside the dome. Considerable reason for the high thermal resistances of bulbs with liquid cooling is the low emissivity of the heat sink surface.

Due to the results based on $\mathrm{FOM}_{\mathrm{R}, \mathrm{A}}$ (see Figure 5), bulb-B with the very high surface temperature and smallest surface area is the most advantageous one. This shows the efficient heat removal based on surface area despite the high thermal resistance. If the system is still operational with a high junction temperature, it can be advantageous to use a small and cheap heat sink and increase its efficiency. Bulb-C has the poorest performance due to its large surface area inside the dome. Since most of the heat sink is inside the dome, heat transfer coefficients are not as high as other bulbs'. Complex heat

Table 3 Weight and surface area of tested lamps

\begin{tabular}{llll}
\hline & Weight $\mathbf{( g )}$ & Surface area $\left(\mathbf{c m}^{\mathbf{2}}\right)$ & Input power (W) \\
\hline Bulb-A & 223 & 173 & 17,0 \\
Bulb-B & 115 & 33 & 9,8 \\
Bulb-C & 227 & 265 & 12,3 \\
Bulb-D & 123 & 104 & 8,7 \\
Bulb-E & 276 & 113 & 7,4 \\
Bulb-F & 273 & 113 & 10,7 \\
Bulb-G & 112 & 149 & 9,4 \\
\hline
\end{tabular}


Table 4 Proposed combinations of FOMs

\begin{tabular}{|c|c|c|c|}
\hline FOM & Formula & Unit & Description \\
\hline$\overline{F O M}$ & $\Delta \mathrm{T} / \mathrm{q}$ & KW & Thermal resistance \\
\hline $\mathrm{FOM}_{\mathrm{R}, \mathrm{m}}$ & $\Delta \mathrm{T} \mathrm{m} / \mathrm{q}$ & $\mathrm{K} \mathrm{kg} / \mathrm{W}$ & Thermal resistance based on mass \\
\hline$F_{R, A}$ & $\Delta \mathrm{T} A / \mathrm{q}$ & $\mathrm{K} \mathrm{cm}{ }^{2} \mathrm{~W}$ & Thermal resistance based on surface area \\
\hline $\mathrm{FOM}_{\mathrm{R}, \mathrm{m}, \mathrm{A}}$ & $\Delta \mathrm{T} m \mathrm{~A} / \mathrm{q}$ & $\mathrm{K} \mathrm{kg} \mathrm{cm}{ }^{2} \mathrm{~W}$ & Thermal resistance based on mass and surface area \\
\hline FOM $L P W$ & $\Phi / P$ & $\mathrm{Im} / \mathrm{W}$ & Luminous efficacy \\
\hline$F O M_{L P W, m}$ & $\Phi / \mathrm{P} \mathrm{m}$ & $\mathrm{Im} / \mathrm{W} \mathrm{kg}$ & Luminous efficacy based on mass \\
\hline FOM LPW,A & $\Phi / P A$ & $\mathrm{Im} / \mathrm{W} \mathrm{cm}{ }^{2}$ & Luminous efficacy based on surface area \\
\hline$F O M_{L P W, m, A}$ & $\Phi / P \mathrm{~m} \mathrm{~A}$ & $\mathrm{Im} / \mathrm{W} \mathrm{kg} \mathrm{cm}{ }^{2}$ & Luminous efficacy based on mass and surface area \\
\hline $\mathrm{FOM}_{\mathrm{T}, \mathrm{L}}$ & $\Delta \mathrm{T} / \Phi$ & $\mathrm{K} / \mathrm{Im}$ & Opto-thermal resistance \\
\hline $\mathrm{FOM}_{T, \mathrm{~m}, \mathrm{~L}}$ & $\Delta \top \mathrm{m} / \Phi$ & $\mathrm{K} \mathrm{kg} / \mathrm{lm}$ & Opto-thermal resistance based on mass \\
\hline $\mathrm{FOM}_{\mathrm{T}, \mathrm{A}, \mathrm{L}}$ & $\Delta \mathrm{T} \mathrm{A} / \Phi$ & $\mathrm{K} \mathrm{cm} \mathrm{cm}^{2} / \mathrm{m}$ & Opto-thermal resistance based on surface area \\
\hline$F O M_{T, m, A, L}$ & $\Delta \mathrm{T} \mathrm{m} A / \Phi$ & $\mathrm{K} \mathrm{kg} \mathrm{cm}^{2} / \mathrm{lm}$ & Opto-thermal resistance based on mass and surface area \\
\hline
\end{tabular}

Subscripts

A, Area $\left(\mathrm{cm}^{2}\right)$.

L, Luminous flux $(\mathrm{Im})$.

LPW, Lumen per watt $(\mathrm{Im} / \mathrm{W})$

$\mathrm{m}$, Mass (kg).

P, Power (W).

$\mathrm{R}$, Thermal resistance (K/W).

$\mathrm{T}$, Temperature $\left({ }^{\circ} \mathrm{C}\right)$.

sink and system structure of bulb-C shows that it is not easily manufacturable. Low heat sink surface emissivity is a reason that as a complete system effectively cooled bulb-A has a high thermal resistance based on surface area. Bulb-B with the highest thermal resistance is again the most advantageous one, when both surface area and mass are taken into consideration. Bulb-D and bulb-G have satisfactory performances based on $\mathrm{FOM}_{\mathrm{R}, \mathrm{m}, \mathrm{A}}$. Bulb-B, bulb-D and bulb-G with the best combined performance based on thermal resistance are the ones which are easy to manufacture as well.

Luminous efficacy is a very useful parameter that indicates total system efficacy including optics and electronics, which is defined as

$$
\mathrm{LPW}=\frac{\Phi}{\mathrm{P}}
$$

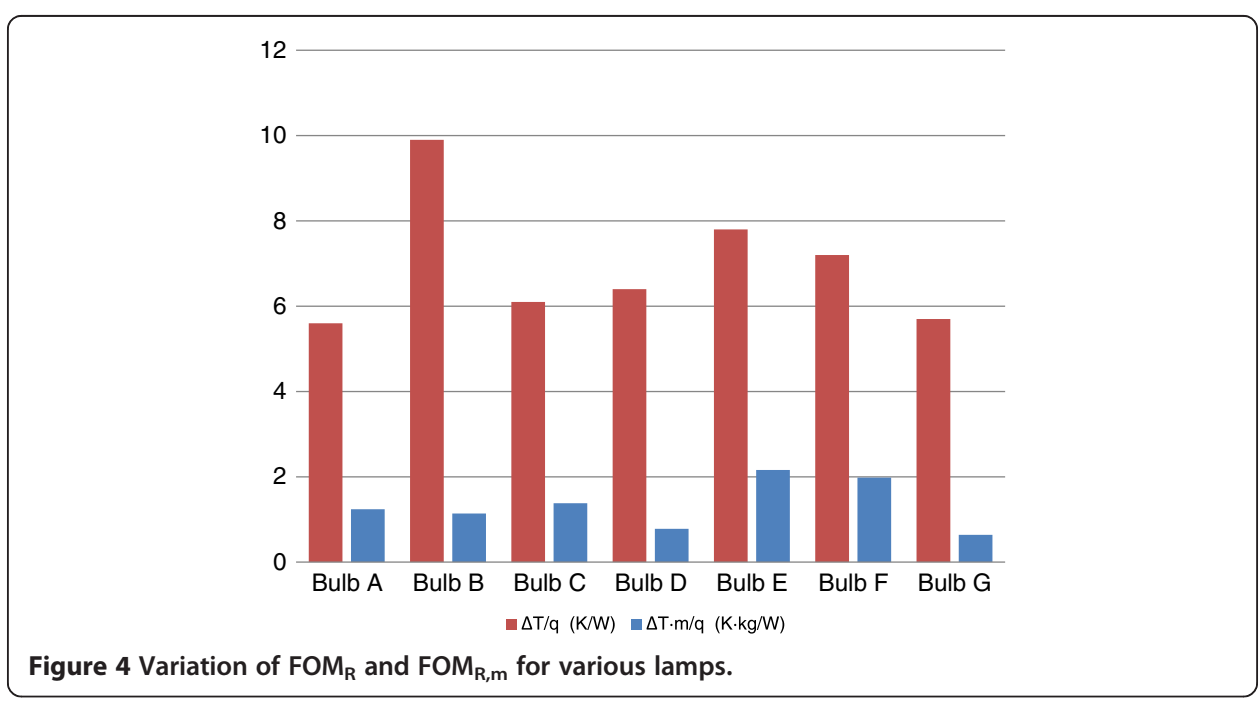




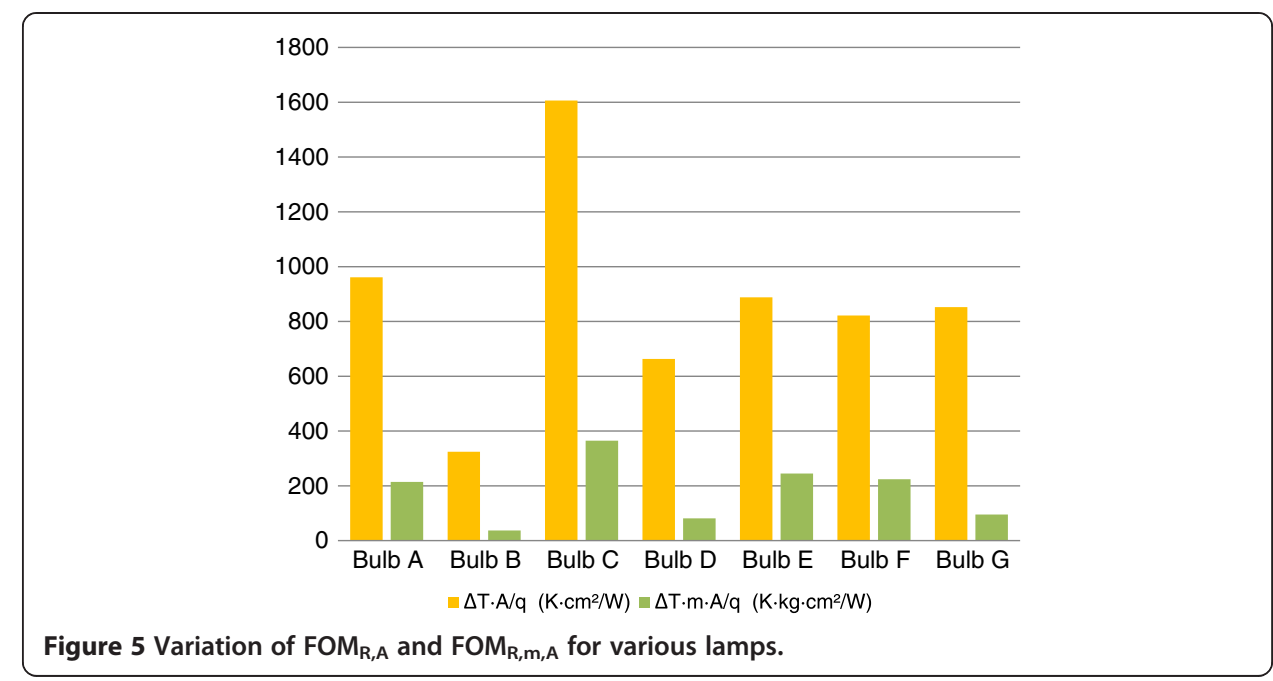

where $\Phi$ represents luminous flux and $\mathrm{P}$ is input power of LED bulb. New FOMs are also produced by the production of luminous efficacy with the inverse of mass and surface area again similar to the FOMs based on thermal resistance. FOM $_{\mathrm{LPW}, \mathrm{m}}$ shows how much lumen is produced per $1 \mathrm{~W}$ input power and $1 \mathrm{~kg}$ bulb mass and $\mathrm{FOM}_{\mathrm{LPW}}$, A shows the efficacy per $1 \mathrm{~cm}^{2}$ surface area of heat sink instead of $1 \mathrm{~kg}$ bulb mass. For a combined efficacy comparison, $\mathrm{FOM}_{\mathrm{LPW}, \mathrm{m}, \mathrm{A}}$ is the ratio of luminous flux to the product of input power, mass and surface area. Luminous efficacy and other system efficacies based on mass and surface area are represented in Figures 6 and 7.

As presented in Figure 6, bulb-B has the highest luminous efficacy. Bulb-B's high performance is due to its efficacy of electrical and optical components, while bulb-G with the second highest luminous efficacy has the lowest maximum heat sink temperature, which is an indicator for a low junction temperature. This emphasizes the importance of power-lumen relation despite the thermal side. Bulb-F with higher input power and higher heat sink temperature has a higher luminous efficacy compared to bulb-E, which also indicates that the thermal performance alone is not adequate for luminous efficacy.

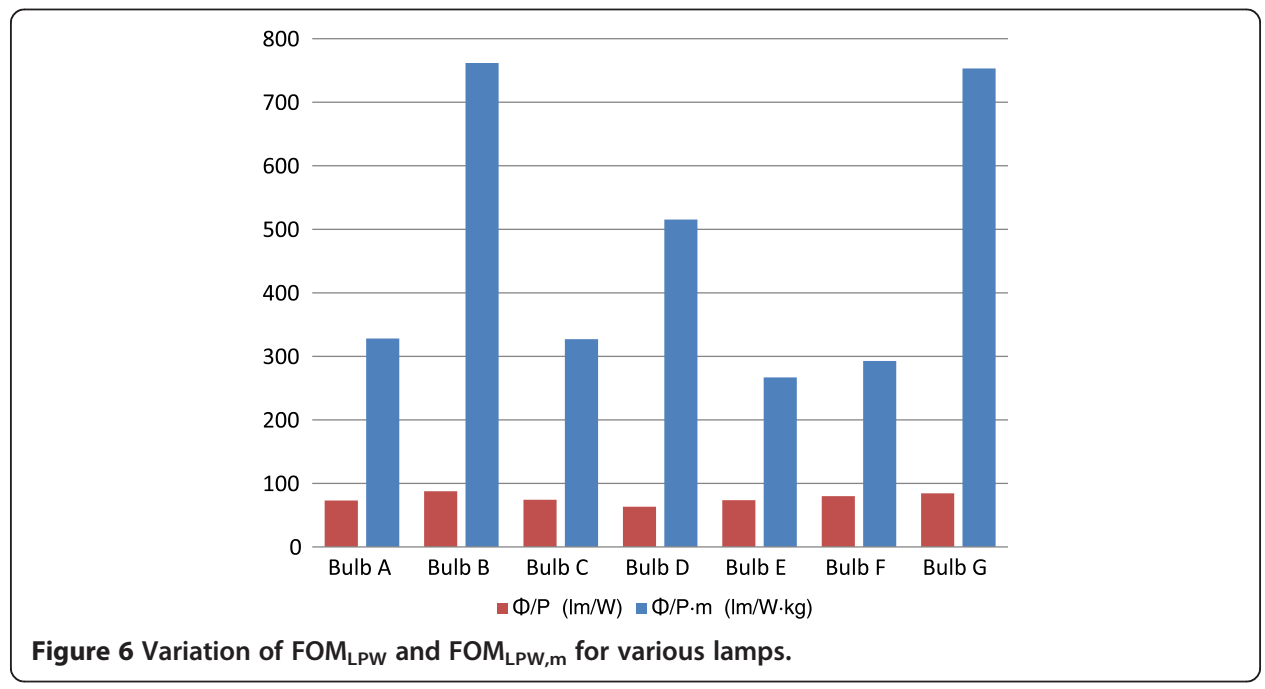




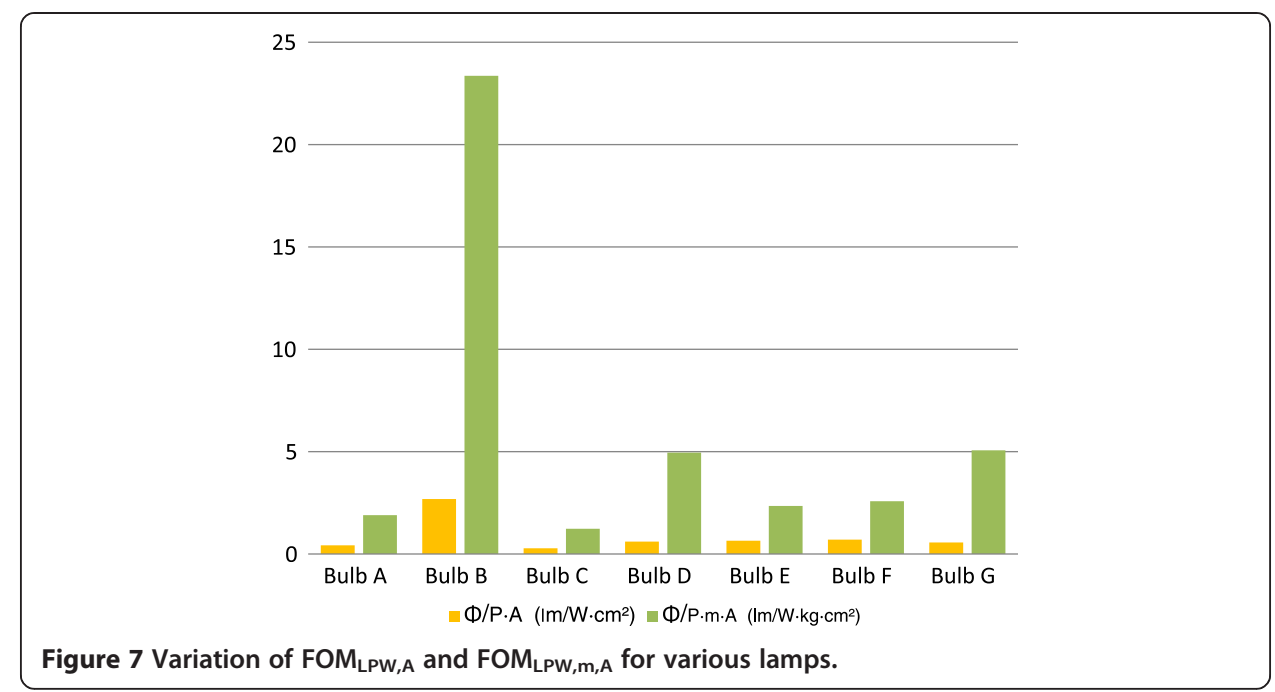

Bulb-B has the best performance again based on mass. Bulb-G with the lowest weight has a very good luminous performance again based on $\mathrm{FOM}_{\mathrm{LPW}, \mathrm{m}}$.

As seen in Figure 7, bulb-B has the highest luminous performance based on surface area. Results show that bulb-G and bulb-D are advantageous based on mass and surface area. Bulb-B has by far the best performance due to $\mathrm{FOM}_{\mathrm{LPW}, \mathrm{m}, \mathrm{A}}$, since it has the highest luminous efficacy with its smallest heat sink and very low weight.

Finally, a new opto-thermal performance indicator is produced offering how much heat sink temperature rises per lumen (see Figure 8). Additional FOMs are also produced by multiplying with mass (see Figure 8), surface area (see Figure 9) to bring various effects together that need to be small against luminous flux. Final FOM (see Figure 9) is the product of all effects.

Figure 8 shows that bulb-G has the best result based on $\mathrm{FOM}_{\mathrm{T}, \mathrm{L}}$ due to its high luminous flux for low temperature rise. Bulb-A and bulb-C have satisfactory opto-thermal performances which are the examples for the combination of smart optical design and

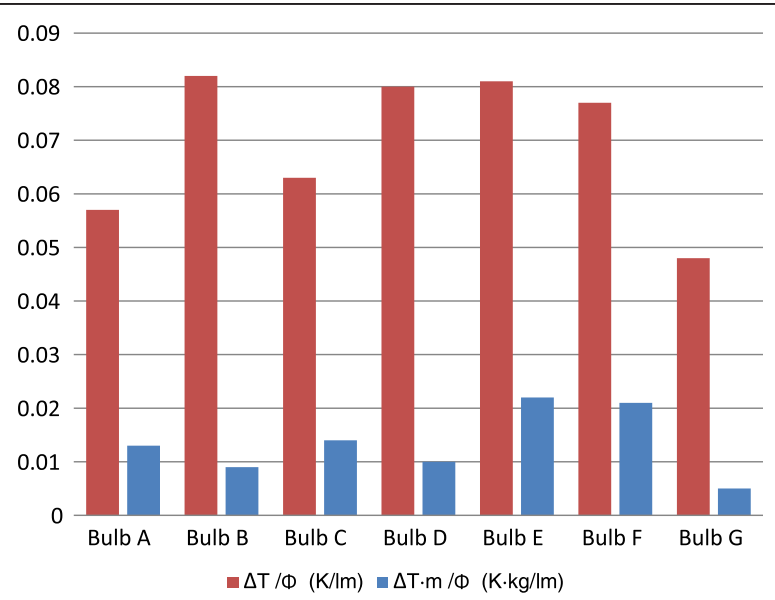

Figure 8 Variation of $\mathrm{FOM}_{\mathrm{T}, \mathrm{L}}$ and $\mathrm{FOM}_{\mathrm{T}, \mathrm{m}, \mathrm{L}}$ for various lamps. 


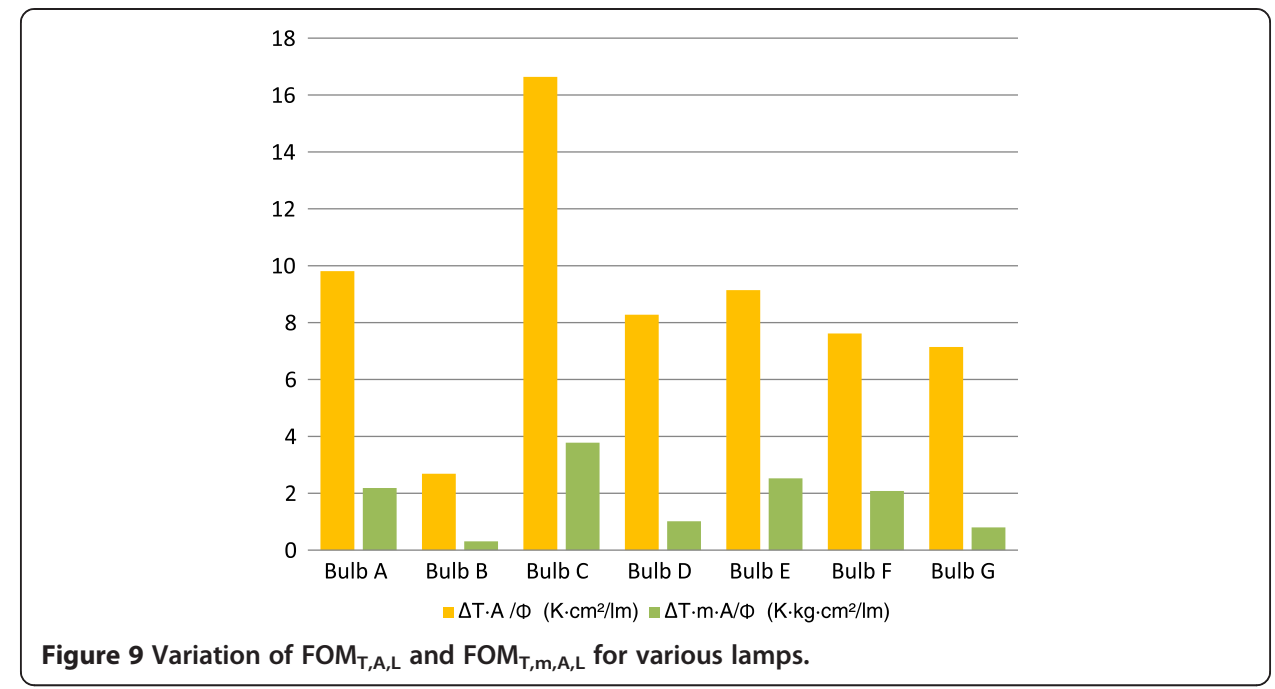

effective cooling. Bulb-F with higher input power has a higher opto-thermal performance compared to bulb-E. Bulb-G's very good performance based on mass is due to its very low weight beside its low opto-thermal resistance. Bulbs B and D have good results based on $\mathrm{FOM}_{\mathrm{T}, \mathrm{m}, \mathrm{L},}$, despite the fact that they have high opto-thermal resistances.

Figure 9 indicates that bulb B has the lowest opto-thermal resistance based on surface area. Bulb-C has the poorest performance based on $\mathrm{FOM}_{\mathrm{T}, \mathrm{A}, \mathrm{L}}$. Results show that bulb- $B$ has the highest opto-thermal performance for its product with mass and surface area. Extra ordinary performance of bulb-B is due to light-weight and small heat sink. Low opto-thermal resistance, small and light-weight heat sinks make G and D other advantageous products from the view of combined effects. Bulb-C has the highest combined opto-thermal resistance due to large surface area and weight.

These newly proposed FOMs help to analyze as many complex aspects together as possible. But some points are not taken into account. Optical consideration includes only the luminous flux, not light quality parameters like CCT and CRI. Bulb-G has the highest CCT, despite the fact that it has satisfying results for manufacturer's and consumer's other primary concerns. Bulb-B with very high efficacy and low combined resistances has very good results; although it has the highest thermal resistance, which normally compromises reliability and life time. However, bulb-B is supposed to have a very long lifetime according to the information provided by manufacturer. It can be explained with the high quality of driver electronics and LEDs. Intricate surface of heat sink is not desired from vendor in common but it does not directly mean that this will bring unacceptable time increase or price jump for the production. It is also possible to make this advantageous, when the lighting manufacturers have access for the development of the production technology. While these FOMs provide a tool to compare various design options, manufacturing and cost of the thermal solution should be considered together. FOMs provide a good general view from many aspects. Some or all of them can be case-based used for a detailed analysis and comparison. It should be remarked that the evaluation method is not only based on cooling approaches or heat sink performances of lamps. In this study total system performances are presented, which is also affected by LED packages, driver electronics, optical components, other 
thermal components and different operating conditions. For a plain performance evaluation of various design approaches of heat sinks, thermal and optical analysis of lamps can be computationally performed based on CAD models under same boundary conditions that may affect above mentioned factors in future studies.

\section{Conclusions}

In this study, a number of FOMs based on thermal resistance, luminous efficacy and newly proposed opto-thermal resistance are presented to offer a collective interpretation of LED lamps. It gives researchers an opportunity to analyze the system on desired performance points and evaluate the system in a restricted area that has to be improved. This is crucial to understand the interaction of design variables. A number of conclusions can be drawn based on the current analysis;

- While thermal performance is crucial for the LED products, large heat sinks or other cooling components with large weights reduce consumer acceptance.

- Generating maximum amount of lumens at the minimum input power and low junction temperature increases total system performance.

- Power-lumen efficiency isolated from temperature dependence is also crucial.

- Developing a novel heat sink with the lowest thermal resistance and with an optimized mass is the key for improving LED systems.

- Efficacy, mass, thermal performance, input power, and heat sink as the key component all impacts LED systems and combined FOMs will help designers and end users.

- Design and cooling approach of the system should not be fixed based on only one aspect; manufacturer's respective manufacturing capability and costs for individual components enable the combinations of primitive and high tech components.

Developed FOMs provide valuable information not only for engineers but also for vendors and consumers to understand LED lamps. While those proposed FOMs carry some insight, they can certainly be expanded by incorporating other performance metrics and they can be improved to find the best-representing parameters for SSL products.

Competing interests

The authors declare that they have no competing interests.

Authors' contributions

MNI carried out the design of the study, performed the experimental analysis and drafted the manuscript. MA participated in design and helped to draft the manuscript. All authors read and approved the final manuscript.

\section{Acknowledgements}

Financial support for this program is provided by EU FP7 CIG program. We thank EU FP7 CIG grant (304013) for the partial support of this research.

Received: 2 March 2014 Accepted: 2 May 2014

Published: 27 May 2014

References

1. Smalley RE: Future Global Energy Prosperity: The Terawatt Challenge. 30th edition. Materials Research Society Bulletin; 2005.

2. U.S. Department of Energy: 2010 Buildings Energy Data Book. 2011.

3. Alvin C, Chu W, Cheng C, Teng J: Thermal Analysis of Extruded Aluminum Fin Heat Sink for LED Cooling Application. Taipei: International Microsystems Packaging Assembly and Circuits Technology; 2011. 
4. Dharma Rao V, Naidu SV, Govinda Rao B, Sharma KV: Combined Convection and Radiation Heat Transfer from a Fin Array with a Vertical Base and Horizontal Fins. San Francisco: World Congress on Engineering and Computer Science; 2007.

5. Bar-Cohen A, lyengar M: Design and Optimization of Air-Cooled Heat Sinks for Sustainable Development, Volume 4 25th edition. IEEE Transactions on Components and Packaging Technologies; 2002.

6. Dogruoz MB, Arik M: On the Conduction and Convection Heat Transfer from Lightweight Advanced Heat Sinks, Volume 2. 33rd edition. IEEE Transactions on Components and Packaging Technologies; 2010.

7. FLIR IR camera manual, 090901 SC5000 Datasheet.

doi:10.1186/2196-1107-1-8

Cite this article as: Inan and Arik: A multi-functional design approach and proposed figure of merits for solid state lighting systems. Journal of Solid State Lighting 2014 1:8.

Submit your manuscript to a SpringerOpen ${ }^{\circ}$ journal and benefit from:

- Convenient online submission

- Rigorous peer review

- Immediate publication on acceptance

- Open access: articles freely available online

- High visibility within the field

Retaining the copyright to your article

Submit your next manuscript at $\boldsymbol{\nabla}$ springeropen.com 